\title{
Bilateral Discrimination of Tactile Patterns without Whisking in Freely Running Rats
}

\author{
(DPauline Kerekes, Aurélie Daret, Daniel E. Shulz, ${ }^{\star}$ and Valérie Ego-Stengel* \\ Unité de Neuroscience, Information et Complexité, Centre National de la Recherche Scientifique, FRE 3693, 91198 Gif-sur-Yvette, France
}

A majority of whisker discrimination tasks in rodents are performed on head-fixed animals to facilitate tracking or control of the sensory inputs. However, head fixation critically restrains the behavior and thus the incoming stimuli compared with those occurring in natural conditions. In this study, we investigated whether freely behaving rats can discriminate fine tactile patterns while running, in particular when stimuli are presented simultaneously on both sides of the snout. We developed a two-alternative forced-choice task in an automated modified T-maze. Stimuli were either a surface with no bars (smooth) or with vertical bars spaced irregularly or regularly. While running at full speed, rats encountered simultaneously the two discriminanda placed on the two sides of the central aisle. Rats learned to recognize regular bars versus a smooth surface in 8 weeks. They solved the task while running at an average speed of $1 \mathrm{~m} / \mathrm{s}$, so that the contact with the stimulus lasted $<1$ typical whisking cycle, precluding the use of active whisking. Whisker-tracking analysis revealed an asymmetry in the position of the whiskers: they oriented toward the rewarded stimulus during successful trials as early as $60 \mathrm{~ms}$ after the first possible contact. We showed that the whiskers and activity in the primary somatosensory cortex are involved during the discrimination process. Finally, we identified irregular patterns of bars that the rats can discriminate from the regular one. This novel task shows that freely moving rodents can make simultaneous bilateral tactile discrimination without whisking.

Key words: freely running behavior; somatosensory cortex; tactile discrimination; whiskers

\section{Significance Statement}

The whisker system of rodents is a widely used model to study tactile processing. Rats show remarkable abilities in discriminating surfaces by actively moving their whiskers (whisking) against stimuli, typically sampling them several times. This motor strategy affects considerably the way that tactile information is acquired and thus the way that neuronal networks process the information. However, when rats run at high speed, they protract their whiskers in front of the snout without large movements. Here, we investigated whether rats are able to discriminate regular and irregular patterns of vertical bars while running without whisking. We found that the animals can perform a bilateral simultaneous discrimination without whisking and that this involves both whiskers and barrel cortex activity.

\section{Introduction}

The rodent whisker system has been a widely used model to study behavioral and neurobiological processes underlying the analysis

Received Feb. 24, 2017; revised May 22, 2017; accepted June 17, 2017.

Author contributions: P.K., D.E.S., and V.E.-S. designed research; P.K., A.D., and V.E.-S. performed research; P.K. analyzed data; P.K., D.E.S., and V.E.-S. wrote the paper.

This work was supported by the Centre National de la Recherche Scientifique, the Human Frontier Science Program Organization (Grant CDA 00044-2010), and the Agence Nationale Recherche (NeuroWhisk). We thank Patrick Parra and Jean-Yves Tiercelin for mechanical design and construction of the maze; Paul Galloux for advice and help concerning the electronics of the maze; Caroline Correia and Hind Baba Aïssa for training rats and the development of the behavioral protocol; and Zuzanna Piwkowska, Thomas Deneux, Evan Harrell, Brice Bathellier, Luc Estebanez, and Isabelle Férézou for advice during the behavioral training and discussions concerning the interpretation of the results.

The authors declare no competing financial interests.

*D.E.S. and V.E.-S. contributed equally to this work.

Correspondence should be addressed to either Daniel E. Shulz or Valérie Ego-Stengel, Unité de Neuroscience, Information et Complexité (UNIC), Centre National de la Recherche Scientifique, FRE 3693, 91198 Gif-sur-Yvette, France.E-mail: daniel.shulz@unic.cnrs-gif.fr or valerie.stengel@unic.cnrs-gif.fr. of surface properties (for review, see Jadhav and Feldman, 2010). In particular, the whisker system allows focusing on the sensorimotor strategy used by the animals during discrimination: can we observe particular features in the motor command when an animal explores a stimulus that would optimize the acquisition of tactile information? For instance, in humans, to feel whether a surface is smooth or rough, the subjects may sweep their fingertips against it, whereas to determine whether an object is vibrating, they may apply immobile fingertips onto it (for review, see Johnson and Hsiao, 1992).

Rodents are able to sweep their whiskers in a rhythmic fashion (whisking) to scan surfaces (Carvell and Simons, 1990; von Heimendahl et al., 2007). Whisking allows sampling the stimuli several times and adjusting parameters such as speed and ampli- 
tude of the movement to improve the information content of the signal (Zuo et al., 2011). Indeed, rats spontaneously use whisking in discrimination tasks involving sandpaper (von Heimendahl et al., 2007) or microgrooved surfaces (Carvell and Simons, 1990). However, rats are also able to analyze aperture size (Krupa et al., 2001) and perform temporal frequency discrimination (Mayrhofer et al., 2013; Miyashita and Feldman, 2013) without overtly moving their whiskers, in a way that has been previously described as a passive reception mode of the tactile inputs (Kleinfeld et al., 2006). Moreover, it has been shown that whisker movements can significantly decrease the performance of head-fixed rats in a vibration detection task (Mayrhofer et al., 2013). These results suggest that whisking might not be required for discrimination of stimuli, but rather appears in laboratory tasks to be a consequence of the experimental conditions. In particular, animals that are head-fixed or restrained on a platform may whisk merely to contact the stimuli. We therefore decided to test whether rats are able to discriminate surface properties without whisking by placing the stimuli in the middle of a long alley so that rats would tend to run past the stimuli rather than stop on them. At full speed, the contact should occur within a duration of less than a typical whisking cycle.

In studies using freely behaving rats, the animals discriminate two stimuli by sampling them with both sides of the snout successively (Carvell and Simons, 1990; von Heimendahl et al., 2007; Morita et al., 2011; Zuo et al., 2011). It is still unknown whether, under these unrestrained conditions, rats could differentiate two stimuli received simultaneously, one on each side. We took advantage of the running alley configuration to test whether animals are capable of bilateral discrimination.

The primary somatosensory cortex (S1) is involved in many whisking-based tasks such as roughness discrimination (GuicRobles et al., 1992) and object localization (O'Connor et al., 2010), but its necessity in tasks without whisking is still debated. Indeed, $\mathrm{S} 1$ is required for detecting oscillations of panels applied onto immobile whiskers (Miyashita and Feldman, 2013), but not for detecting frequency changes of air puff pulses (Hutson and Masterton, 1986), although this is still controversial (Sachidhanandam et al., 2013). To test the effects of S1 silencing in tactile discrimination, we designed a cranial implant for chronic application of the GABAergic agonist muscimol.

For this study, we developed a novel, two-alternative forced choice task inciting the rats to discriminate stimuli by running past them at high speed, sampling each stimulus with whiskers on one side of the snout and only once. Rats learned to discriminate a surface with a series of vertical bars regularly spaced versus a smooth surface. They could also discriminate an irregular series from the regular one. The stimulus sampling occurred in $<100 \mathrm{~ms}$ and the animals oriented their whisker arrays toward the rewarded stimulus as soon as $60 \mathrm{~ms}$ after the first possible contact. Both whiskers and S1 activity were involved during the discrimination process.

\section{Materials and Methods}

Animals. All experimental and surgical procedures were approved by the French Ethical Committee (project \#526.01). In total, 10 male adult Long-Evans rats were used. Two animals were used exclusively for acute electrophysiology experiments and eight animals were trained on the discrimination task. The training began when the animals were 6 weeks old and weighed $250-350 \mathrm{~g}$. The rats were housed individually in cages of length $60 \mathrm{~cm}$ and width $44 \mathrm{~cm}$ with a 25-cm-long tunnel to enrich their environment and were food deprived to $80-85 \%$ of their normal weight during the whole learning course. The $100 \%$ weight reference was deter- mined with two control rats that were housed in the same conditions and fed ad libitum.

Behavioral apparatus. Freely moving animals had to discriminate between pairs of $10.2 \mathrm{~cm}$ long surfaces, with either 18 vertical bars spaced irregularly, 18 vertical bars spaced regularly, or no vertical bars (smooth). The stimuli were designed using the SolidWorks software and 3D printed (Easy Up 120 printer; A4 Technologie) using black ABS plastic material. For the regular series of bars, the interval between the bars was $3.9 \mathrm{~mm}$ wide and the bars were $2 \mathrm{~mm}$ thick and $2 \mathrm{~mm}$ wide (see Fig. 1A1). The two surfaces were facing each other in the central alley of the maze. The stimuli holders were $25-\mathrm{cm}$-wide rotating hexagons with a stimulus on each of the six sides. We always used three different copies of the same stimulus to avoid learning specific details of one particular surface. The stimuli combinations were pseudorandomly distributed across trials. The maze (Fig. 1A1) was automated with a custom-made program implemented on an Arduino Mega2560 board with servo motors to move the doors, stepper motors to rotate the stimuli holder, two pellet dispensers (Campden Instruments 80209) controlled by TTL inputs and four infrared (IR) sensors (Adafruit product ID 2167). The sensor signals were used to trigger the different trial events: open/close the doors, change the stimuli, deliver the rewards, and provide a measure of the running speed in the central alley. At the beginning of learning, we used copies of the rewarded stimulus mounted on servo motors as reminders (R1 and R2). The reward was two $45 \mathrm{mg}$ pellets (Bilaney F0021) per successful trial and was triggered by the IR sensor placed $2 \mathrm{~cm}$ in front of the reward cup. Auditory masking noise (white noise) was presented with a loudspeaker located $50 \mathrm{~cm}$ away from the maze. We cleaned the maze after each session with a $30 \%$ ethanol solution to mask olfactory cues and checked after learning that the animals were not using olfactory cues from the stimuli themselves. All training took place in the dark to avoid visual cues. The position of the rewarded stimulus, the choice, and the speed of the animal in the central alley were automatically recorded through the Arduino interface for each trial.

Behavioral protocol. The goal of the learning protocol was to have the rats discriminate between the surface with the regular series of bars and another surface, the smooth one first, while running at high speed. During the first week, the animals were habituated to be handled and taken to the experimental room in the dark with the white noise and maze motor noise. The animals were separated in individual cages on the third day and food restriction began on the sixth day. On day 7, rats were placed in each arm of the maze with all doors closed, first during $2 \mathrm{~min}$ in the light, then during $2 \mathrm{~min}$ in the dark. They received one pellet in the dispenser cup each time they crossed the IR sensor to make them associate this site with the reward. The day after, we began the learning of the association between the regular bars surface and the reward. This learning course was divided into three stages with one 25-min-long session per day. During the first stage, in addition to the main stimuli in the central alley, we used reminders of the rewarded stimulus (Harvey et al., 2012) before the choice point and just before the reward site (R1 and R2, respectively; Fig. $1 A 2)$. The rats had to turn to the side corresponding to the regular bars surface to get a reward and were forced to continue forward in the maze because the doors closed automatically just after they went through. The animals had to perform at least $80 \%$ correct trials during three consecutive sessions to enter the next stage. During the second stage (Fig. 1A2), only the reminder before the choice point was presented (in addition to the main stimuli). The same criterion as for stage 1 was used to consider this stage completed. Throughout the third and last stage (Fig. 1A2), the reminders at the choice site were displayed during the first five trials and removed for the rest of the session. The rats had then to learn the task with the main stimulus site only. For this stage, the performance throughout learning was quantified on all trials except the first five ones. The criterion to complete the third stage was to reach a mean performance at $\geq 70 \%$ and a SD $\leq 4.2 \%$ on 6 consecutive sessions. This threshold for the SD value was determined by calculating the mean SD on seven blocks of six sessions on two rats trained in a preliminary experiment. More precisely, these rats were initially trained with an empty space facing the regular series and the smooth surface was introduced during stage 3 only. These two animals are not included in the results of Figure 2 
A1

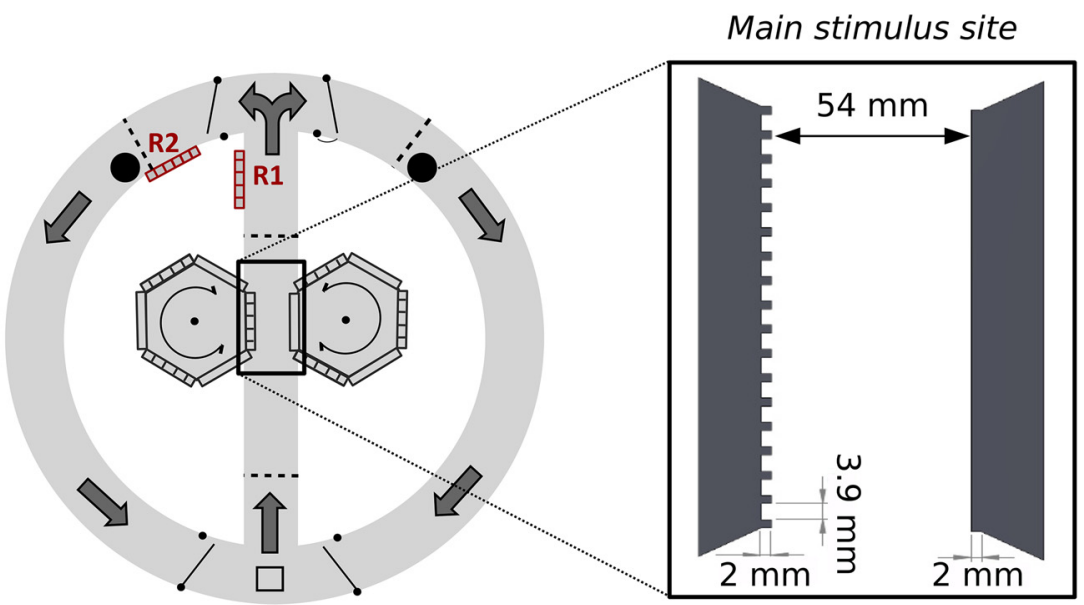

\begin{tabular}{|l|l|l|l}
\hline & & & \\
\hline
\end{tabular} Rewarded stimulus

$\square$ Non rewarded stimulus

Reward site

R1, R2 Reminders displayed on

- ... IR detectors

the rewarded side

$\square \quad$ Starting point of each trial

\section{A2}

Behavioral protocol
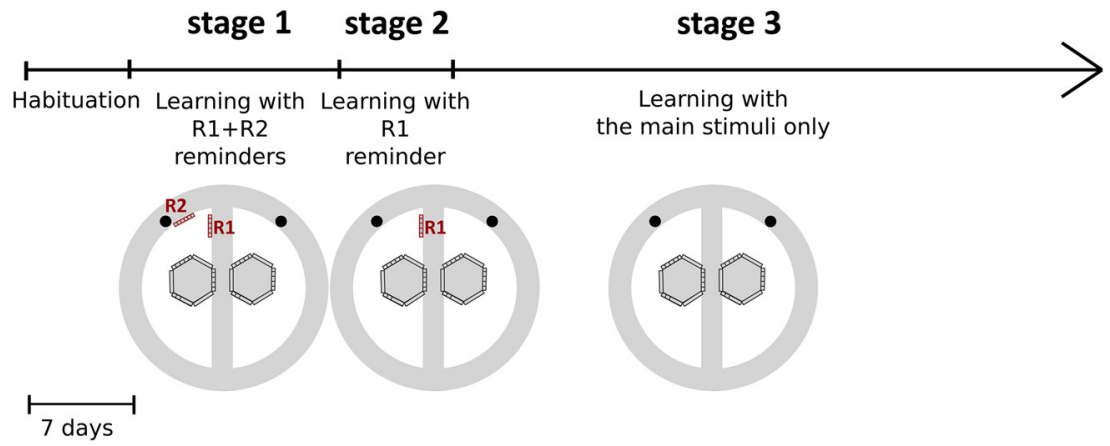

B

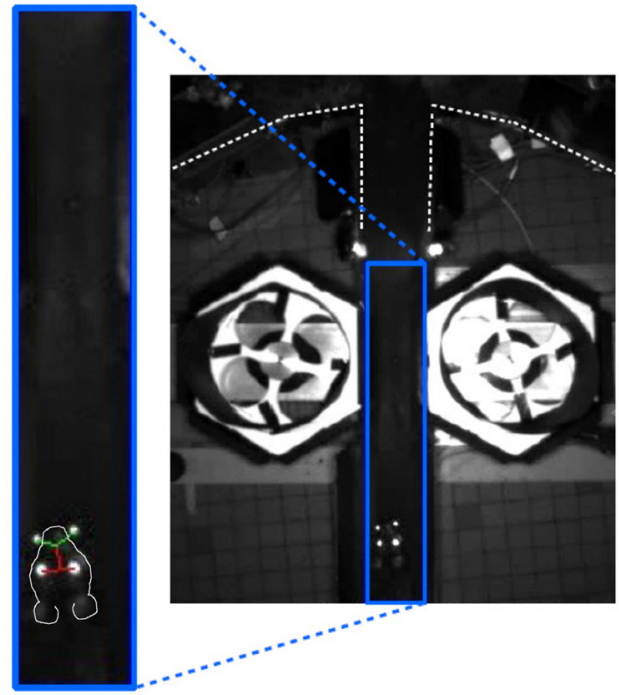

C
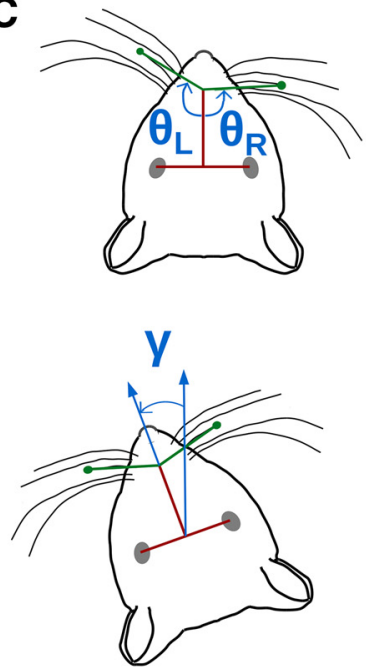
A

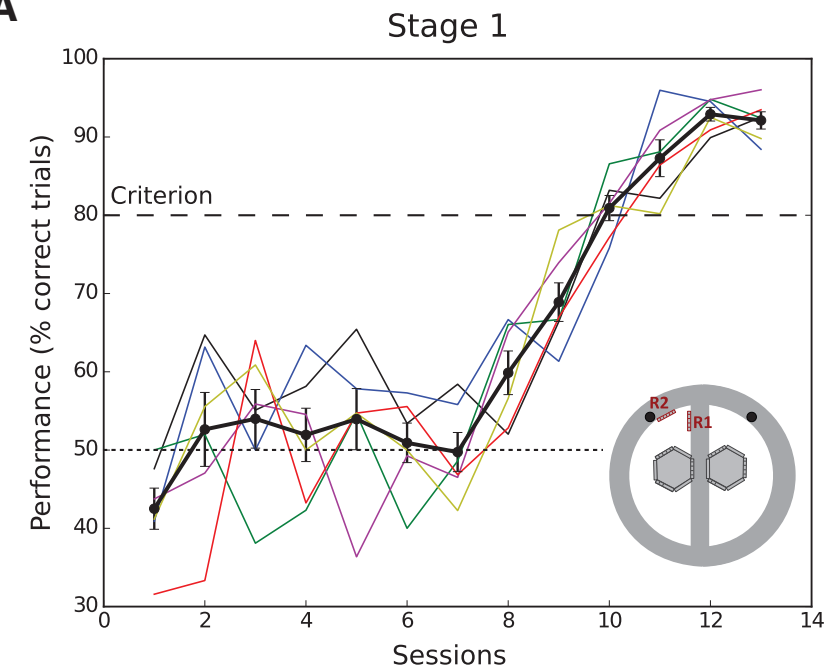

B

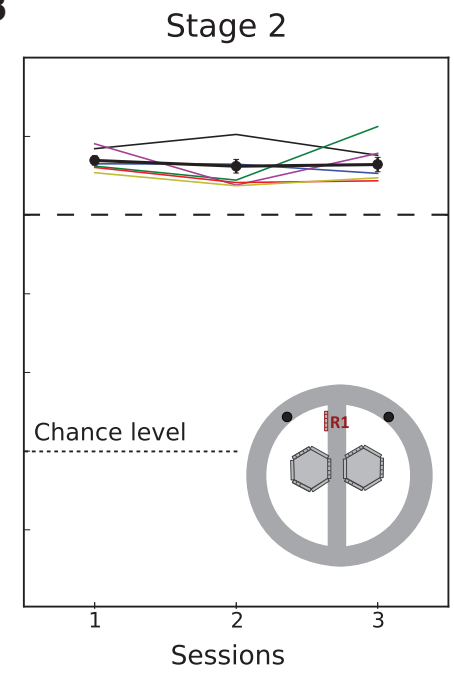

C

Stage 3

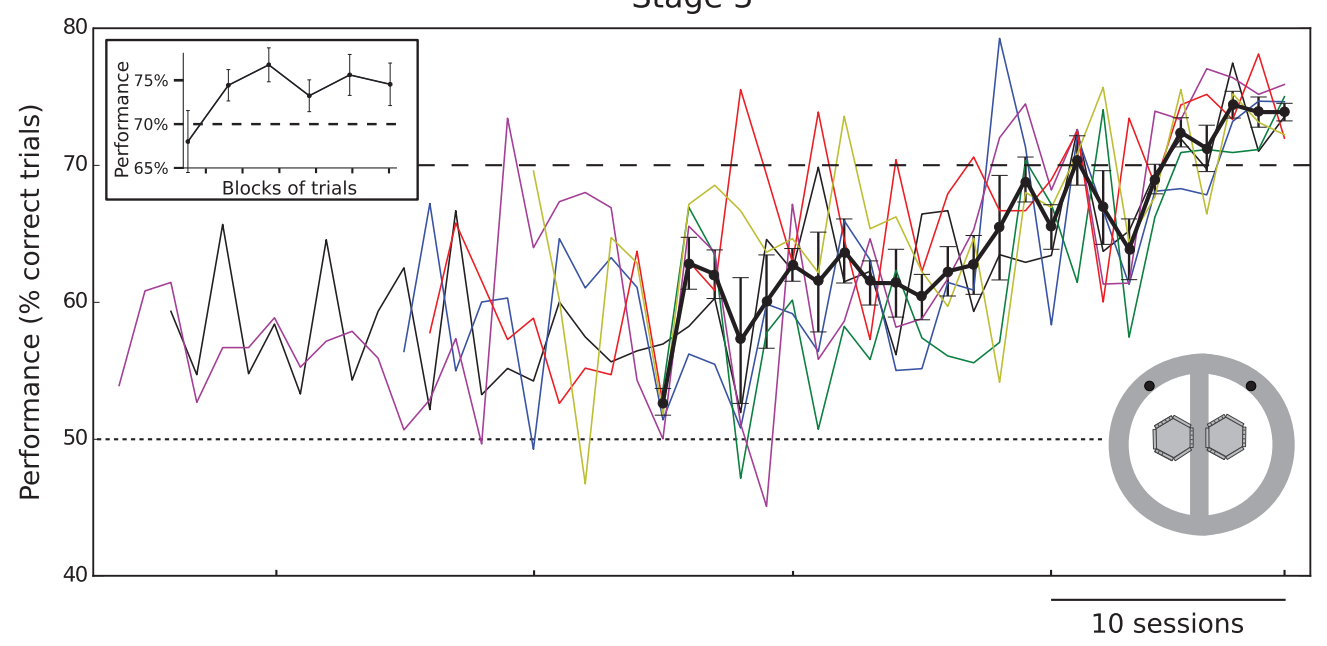

Figure 2. Discrimination between a smooth surface and a surface with regularly spaced vertical bars in 8 weeks. $A$, Learning curves for stage 1 with two reminders of the rewarded stimulus. The animals had to achieve at least $80 \%$ of correct trials during three consecutive sessions to enter the following stage. $\boldsymbol{B}$, Learning curves with one reminder at the choice position (same criterion as for stage 1). C, Learning curves for stage 3, with only the main stimuli present in the central alley. The criterion to complete learning was to maintain a mean performance of $70 \%$ with a maximal SD of $4.2 \%$ on 6 consecutive sessions. All curves have been aligned on the final session of learning for each rat (range $25-46$ sessions). The inset details the performance within a session, calculated by splitting each session into 6 blocks of trials of equal length and averaged over the last 6 sessions of stage 3 ( $n=6$ rats). In this figure and the following, the thick dark line indicates the mean performance \pm SEM and color traces show individual results.

$\leftarrow$

Figure 1. Behavioral maze and learning protocol for investigating whisker-based discrimination in the freely moving rat. $\boldsymbol{A} \boldsymbol{1}$, Schematic representation of the automated maze. For each trial, the rats had to run in the central alley through the main stimulus site (black rectangle, enlarged on the right). A smooth surface and a surface with a regular series of bars were displayed on the right and left sides randomly. At the end of the alley, the animals had to turn to the side on which the regular series of bars was presented to receive a reward. $\mathbf{A 2}$, After a period of habituation, task learning was divided into several stages. In stage 1 , two reminders of the rewarded stimulus were displayed on the rewarded side: one at the end of the alley near the choice point (R1) and the other next to the reward site (R2). During stage 2, reminder R1 was displayed only. During stage 3 , only stimuli at the main site were present. $\boldsymbol{B}$, Top view of a rat running in the alley before contacting the stimuli. The blue rectangle encloses the tracking area. The corresponding enlargement shows tracking of the head direction using IR light reflection on the eyes (red lines) and tracking of the angle values of the right and left whiskers carrying reflection material (green lines). C, Variables measured during tracking. Top, Absolute angle of the right and left whiskers (respectively, $\theta_{R}$ and $\theta_{L}$ ) relative to the head axis. When the whiskers are oriented toward the right side, $\theta_{R}<\theta_{L}$ and the ratio is $<1$. Bottom, Head direction $(\gamma)$ relative to the central alley (vertical on the video frames as in $\boldsymbol{B}$ ). and were only used for control experiments (whisker cutting and cortical inactivation; see Fig. 4). Their performance in control sessions, once learning was completed, was indistinguishable from the main group of rats.

Discrimination of the regular and irregular bar series. These experiments were performed on rats that had already learned the regular versus smooth discrimination. Our next goal was to assess whether these rats could report a difference between a regular and an irregular series of bars. We designed five types of irregular series, all containing 18 bars, that could be perceived as intermediate stimuli between the smooth surface and the regular series (Fig. $3 A$ ). One irregular series was designed by picking the location of the bars at random on the surface (Poisson-like series, containing intervals of $0.5 \mathrm{~mm}$ to at most $13 \mathrm{~mm}$ between 2 bars). The four remaining irregular series included one forced interval between two bars of 30,40,48, or $50 \mathrm{~mm}$ placed randomly and smaller intervals between the remaining bars. We hypothesized that this wide smooth interval would be a feature similar to the smooth stimulus and consequently that, the wider this interval, the more easily the rats could discriminate the irregular series from the regular series. Between two consecutive tests involving an irregular series, the rats were trained on the regular versus smooth paradigm for at least $4 \mathrm{~d}$. For each type of irregular 
A

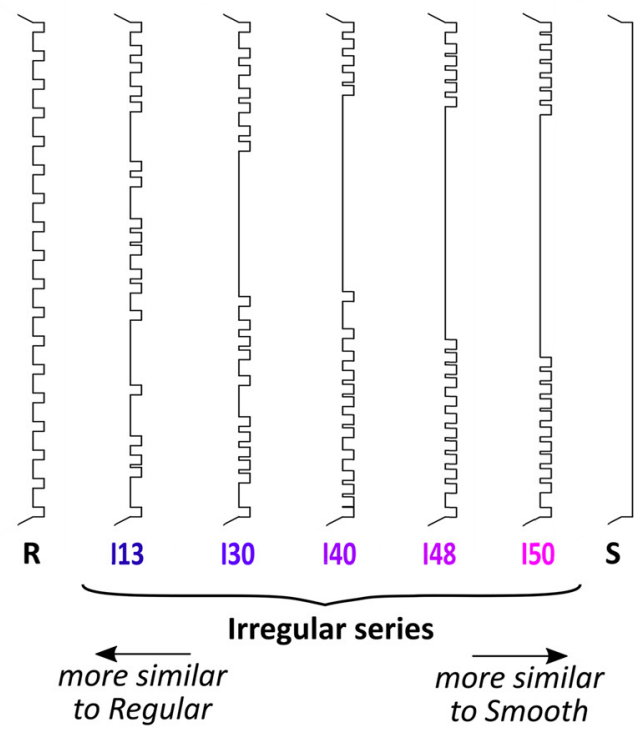

B

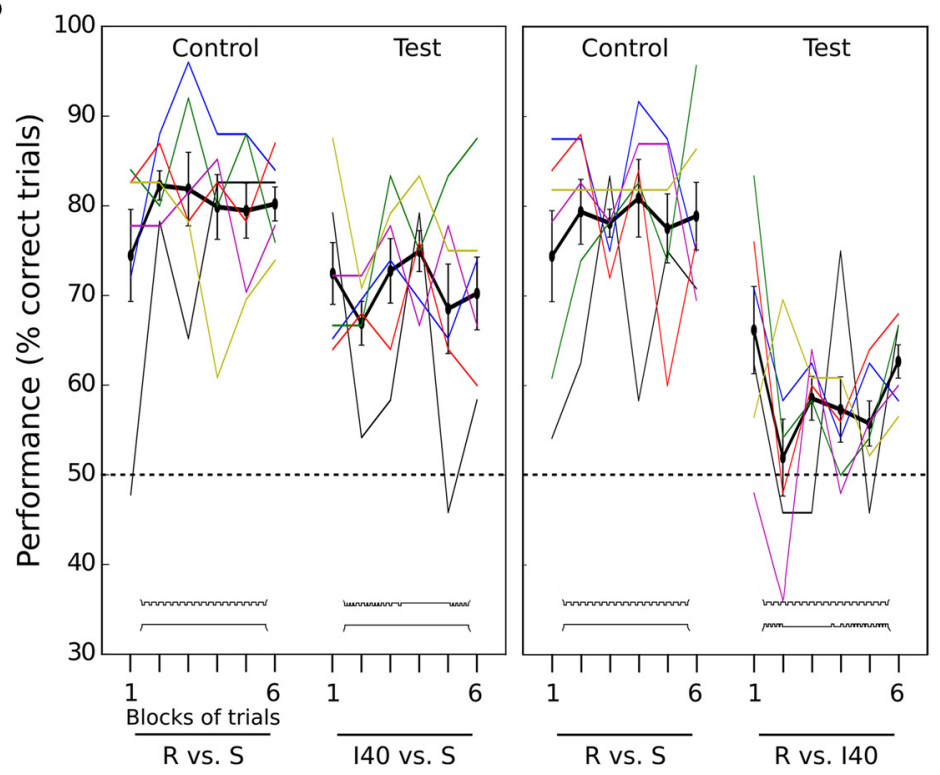

C

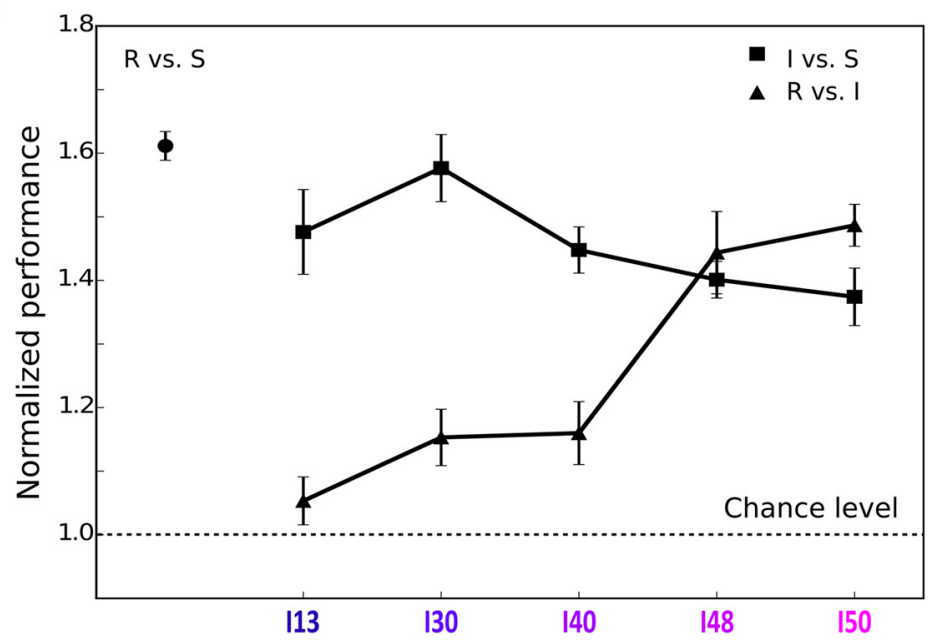

Figure 3. Discrimination of surfaces with irregularly spaced bars from the smooth and regular stimuli. $A$, Profiles of the irregular series arranged by increasing width of the largest interval between bars. $\boldsymbol{B}$, Replacement of either the regular or the smooth series, we performed two tests, always in the same order: (1) irregular versus smooth with the irregular stimulus being rewarded and (2) regular versus irregular with the regular stimulus being rewarded.

Whisker cutting. We anesthetized the rats with isoflurane $(3 \%)$ for $2-5 \mathrm{~min}$ and cut all large facial whiskers (rows A-E, arcs 1-7, and the four straddler whiskers) on both sides of the snout with a small pair of scissors. The fur on the face and the microvibrissae were not cut. The animals $(n=7)$ were tested at least $1 \mathrm{~h}$ after the end of the anesthesia.

Surgical procedures. We tested the effects of muscimol application on neuronal activity in the barrel cortex and on behavioral performance in the discrimination task. The animals were anesthetized with isoflurane $(1.5-3 \%$ in $0.2 \mathrm{~L} / \mathrm{min}_{2}$ and $0.8 \mathrm{~L} / \mathrm{min} \mathrm{N}_{2} \mathrm{O}$; Medical Supplies and Services). Their temperature was monitored with a rectal probe and maintained at $37^{\circ} \mathrm{C}$ with a heating blanket. The respiration was monitored throughout the experiment by means of a piezoelectric sensor placed between the chest and the platform on which the animal rested. After the animal was mounted in a stereotaxic frame, different craniotomies were drilled according to the type of experiment. We performed acute electrophysiological recordings of the neuronal activity during S1 muscimol application ( $n=2$ naive rats) and chronic implantations for muscimol applications during sessions of the behavioral task ( $n=3$ trained rats). For acute experiments, we made craniotomies over S1 $(0-2 \mathrm{~mm}$ posterior, $4-6 \mathrm{~mm}$ lateral from bregma; Paxinos and Watson, 2009) and the hippocampus $(2.6-5.6 \mathrm{~mm}$ posterior, $2.4-5.4 \mathrm{~mm}$ lateral from bregma) on the left side. The two craniotomies joined with a third one between (1.7-4.7 $\mathrm{mm}$ posterior, $4-6 \mathrm{~mm}$ lateral from bregma) to apply muscimol. We used 3D-printed implants with one hole to apply the muscimol on top of the S1 cortex and guiding structures to hold tetrode microdrives. For chronic implantations of trained animals, bilateral craniotomies were made over $\mathrm{S} 1$ (0.4-4 mm posterior, $4-6 \mathrm{~mm}$ lateral from bregma). These experiments were performed using 3D-printed implants with holes to apply the muscimol on top of the S1 cortex bilaterally. For both chronic and acute implantations, the dura was removed in the craniotomies and the implant was cemented on the skull. Two screws were inserted in the bone to better interlock the implant and the skull with dental cement for chronic experiments and one ground screw was added for acute recordings. At the end of

$\leftarrow$

stimulus by the irregular 140 stimulus ( $n=6$ rats). In both panels, the pretest session (day before replacement) and the test session (day of replacement) are shown, split into six blocks of trials of equal length (20-28 trials depending on the animal). C, Performance divided by chance level after replacement of either the regular (square symbols) or the smooth (triangles) stimuli by each of the irregular series (I13, I30, I40, 148 , and 150). The regular versus smooth performance in this figure was calculated as the mean performance during all pretest sessions. 
chronic implantations, $0.1 \mathrm{ml}$ of saline was added in each reservoir of the implant to protect the craniotomies from getting dried out and a cap of silicon sealant (Kwik-Cast; World Precision Instrument) was used to close the two reservoirs. One hundred microliters of Metacam (meloxicam, $2 \mathrm{mg} / \mathrm{ml}$ ) were injected under the skin in the neck for pain management. Drops of betadine were applied on the skin around the implant to prevent infection.

Cortical inactivation with muscimol. Muscimol hydrobromide powder (Sigma-Aldrich, 18174-72-6) was diluted in PBS and applied to the surface of the cortex (Higley and Contreras, 2006). For acute experiments, we deposited $0.2 \mathrm{ml}$ of muscimol solution $(0.5 \mathrm{mg} / \mathrm{ml})$ in the reservoir using a syringe. For chronic experiments, we anesthetized the animal with isoflurane $(1.5-3 \%)$ to rinse the craniotomies and apply the same muscimol solution. The animals $(n=3)$ were tested on the behavioral task 70 min after muscimol application, thus $\sim 60$ min after the anesthesia ended. After each muscimol session, the animal was anesthetized again for $20 \mathrm{~min}$ to rinse the craniotomies and to apply $0.1 \mathrm{ml}$ of saline solution in each reservoir. In control sessions, we followed the same protocol with saline instead of muscimol.

Electrophysiological recording and whisker stimulation. Neural activity was recorded extracellularly using tetrodes $(20 \mu \mathrm{m} \mathrm{NiCr}$ wire insulated with Teflon; Kanthal Palm Coast). The 3D-printed implants were designed to hold microdrives that guided the tetrodes into the brain (250 $\mu \mathrm{m}$ discplacement per screw turn). Before the implantation, tetrodes were gold plated to decrease their impedance level to $250 \mathrm{k} \Omega$ and to increase the signal/noise ratio. For whisker stimulation, we used a stimulator composed of 25 independent piezoelectric actuators adapted to the five rows and the five caudal arcs of the whisker pad (Jacob et al., 2010). Whiskers were trimmed to a length of $10 \mathrm{~mm}$ and inserted $3 \mathrm{~mm}$ into short polypropylene tubes glued on the actuators. The actuators were driven with RC-filtered (time constant $=2 \mathrm{~ms}$ ) voltage pulses of $30 \mathrm{~ms}$ duration ( $10 \mathrm{~ms}$ rise, $10 \mathrm{~ms}$ hold, $10 \mathrm{~ms}$ fall time) to produce displacements of $0.93^{\circ}$ along the rostrocaudal axis. For our experiments, we used sparse noise stimulation applied on the 24 whiskers. Every sequence of stimulation included the deflection of each of the 24 whiskers in both rostral and caudal directions in a random order at $20 \mathrm{~Hz}$.

Global whisker movement tracking. To track the global movement of the whiskers during behavioral sessions, we glued square IR reflectors $\left(2.25-3 \mathrm{~mm}^{2}, 1-2 \mathrm{mg}\right)$ on the $\mathrm{C} 1$ or $\mathrm{C} 2$ whisker on both sides of the snout at a distance of $8-10 \mathrm{~mm}$ from the follicle. The part of the whisker extending past the reflector was cut so that the whisker could not touch the stimuli. Similar physical loading was tested in a previous study aiming at precise quantification of the kinematics of an individual whisker during contact and was found to not alter the whisker movements (Bermejo et al., 1998). In our conditions, we also verified that possible brushing of the immediately anterior whisker on the reflector did not affect the tracked whisker movement. Importantly, there was no change in performance in the tactile discrimination task after gluing of the reflectors (mean performance \pm SD just before: $79.3 \pm 2.5 \%$ vs just after: $76.5 \pm$ $6.6 \%$, paired $t$ test $p=0.6, n=3$ rats). Note that all sessions described in Figures 2, 3, 4, and 5 were performed with intact whiskers (except in the whisker-cutting sessions) and without markers. Videos were acquired with an IR camera with LED illumination (OptiTrack V100:R2; Blackrock Microsystems) and the NeuroMotive software (Blackrock Microsystems; Fig. 1B). The frames were captured at $100 \mathrm{~Hz}$ with a resolution of $640 \times 480$ pixels. The rat eyes were naturally reflecting the IR beams of the camera (Fig. 1B, blue enlargement). This frame rate and bird's eye view of the setup allowed us to follow the rat head and the global position of the whisker arrays in time, including the detection of whisking $(5-12 \mathrm{~Hz})$. Note that it would not have been sufficient to track individual whiskers precisely in time and space. The tracking was implemented in a custommade Python program using the OpenCV module for image processing and the minEnclosingCircle function to extract the coordinates of the eyes and whisker markers. We analyzed the head direction $\gamma(t)$, the angle of the labeled whiskers $\theta_{\mathrm{R}}, \theta_{\mathrm{L}}(t)$ with the head axis (Fig. 1C), and the speed of the animal before and during stimulus contact. We estimated the time of first possible contact with the stimulus based on the maximal protraction of whiskers relative to the nose $(39 \pm 2.3 \mathrm{~mm}$ mean $\pm \mathrm{SEM}$; Morita et al., 2011).
A few video recordings were performed during the muscimol and whisker-cutting sessions without reflector markers. Three such sessions were tracked manually by clicking on the rat snout and the midpoint between the eyes on $\sim 30$ frames per discrimination trial while the rat was running past the stimuli. For each trial, we extracted the minimal distance from the snout to the stimuli (precision 1 pixel $=1.5 \mathrm{~mm}$ ), the maximal head angle (precision around $4^{\circ}$ ), and the minimal distance from the midpoint between the eyes to the stimuli (precision 1 pixel). We estimated that, when this last measure was $<15 \mathrm{~mm}$, there was a possibility that the head fur was touching the stimuli.

Statistics. Statistical errors are SEM unless indicated otherwise. A binomial test was used to compare behavioral performance to chance level after the following manipulations: whisker cutting, cortical inactivation, and stimuli replacement. All statistical tests were built-in functions from the scipy.stats module of Python.

\section{Results \\ Discrimination of a smooth surface versus a regular series of bars}

We trained six rats to discriminate between two surfaces placed on the right and left sides randomly while running in an alley (Fig. $1 A)$. One surface displayed a regular series of vertical bars and the other one was smooth. The animals had to turn to the side corresponding to the regular stimulus at the end of the alley to obtain a reward. In preliminary tests, we found that the rats had difficulties to learn the task if only the stimuli in the center of the alley were present. Therefore, we added reminders of the rewarded stimulus along the way from the main stimulus site to the reward site (Harvey et al., 2012). During the first learning stage, two reminders were displayed (Fig. $1 A, \mathrm{R} 1$ and $\mathrm{R} 2$ ). The six rats reached the $80 \%$ criterion in 13 sessions ( $\sim 1560$ trials; Fig. $2 A)$. During this stage, the number of trials increased from $20.2 \pm 3.7$ to $129 \pm 18$ trials per session (mean $\pm \mathrm{SD}$ ). The running speed in the central alley increased to $118.7 \pm 7.2 \mathrm{~cm} / \mathrm{s}$ (mean $\pm \mathrm{SD}$ ), confirming that the rats ran at full speed when passing the central stimuli. In the second learning stage, the reminders next to the reward sites (R2) were removed. The high level of performance already during the first session of stage $2(86.9 \pm 3.6 \%$, mean \pm $\mathrm{SD}$ ) indicated that the rats could immediately solve the task without the $\mathrm{R} 2$ reminders (Fig. $2 B$ ). Figure $2 C$ displays the learning curves of the six rats during the last stage of the protocol, when only the main stimuli in the central alley were present. Although there was a large variability from one session to the next, the animals took on average $35.7 \pm 8$ sessions (mean $\pm \mathrm{SD}$ ) to reach the $70 \%$ correct trials level and stabilize their performance (Fig. $2 C)$. The inset in Figure $2 C$ shows the temporal profile of the performance within a session at the end of learning. Typically, the performance started at $\sim 70 \%$ during the first trials, increased, and then stabilized or decreased slightly throughout the session. After learning the whole protocol, the mean running speed was still very high at $117.9 \pm 7.6 \mathrm{~cm} / \mathrm{s}$ (mean $\pm S D$, calculated over 20 sessions after learning, $n=5$ rats). Overall, we conclude from these data that rats can learn to discriminate a smooth surface from one displaying regular vertical bars while freely running at full speed in an alley. The total training course (stages 1-3) lasted for $8.4 \pm 1.6$ weeks (mean $\pm \mathrm{SD}$ ).

\section{Discrimination of regular versus irregular series of bars}

One initial goal of our study was to assess whether rats can discriminate surfaces displaying regular versus irregular patterns of bars. We designed several irregular patterns, all containing 18 vertical bars as the regular series but differing by the locations of these bars. Each series was identified by the widest interval it contained: 13, 30, 40, 48, or 50 mm (I13-I50; Fig. 3A; see Mate- 
rials and Methods). First, we tested whether, after having learned the regular versus smooth discrimination task, the rats could perceive the sudden replacement of the regular surface by an irregular surface. If this was the case, then we would expect a drop in performance after the replacement. Indeed, Figure $3 B$, left, shows the results of a standard regular versus smooth discrimination session, followed on the next day by a test session in which the regular series was replaced by the irregular I40 series. In the control session, the performance was $\sim 75 \%$ during the first block and then tended to increase, as was typical of regular versus smooth sessions (Fig. 2C, inset). During the test session, the performance started almost at the same level, but then never increased. This altered performance in the I40 versus smooth session (paired $t$ test compared with regular vs smooth, $p<0.05$, $n=6$ ) indicates that the animals detected a change in the surface displaying a bar pattern, which led them to choose the side corresponding to the nonrewarded smooth surface more often than in control sessions.

We then investigated whether the rats could discriminate directly the regular series versus the I40 series. In those sessions, the performance of three rats of six was significantly above chance (paired $t$ test, $p<3 \times 10^{-3}$; Fig. $3 B$, right). In fact, in the first block of trials, the rats made the right choice in $65 \%$ of the trials, confirming that they discriminated the two surfaces. We hypothesized that the long smooth interval present on the I40 surface was the main feature that led the rats to treat the I40 surface as a smooth one when performing correctly in these sessions. Conversely, the 18 bars present on the I40 surface led the rats to treat it as equivalent to the regular surface in the I40 versus smooth session. The mean performance was significantly higher during the I40 versus smooth discrimination than during the regular versus I40 one (paired $t$ test, $p<7 \times 10^{-3}, n=6$ ), suggesting that the rats perceived the I40 as more similar to the regular than the smooth surface.

More generally, we repeated these tests with other irregular patterns of bars and observed that the regular versus irregular task performance increased with the size of the maximal interval within the irregular series. Conversely, the irregular versus smooth task performance decreased when the size of the maximal interval increased (Fig. 3C). Particularly, the I48 versus smooth and the regular versus $\mathrm{I} 48$ tasks resulted in a similar level of performance. This indicates that, from a perceptual point of view, the I48 may be equally close to the smooth as to the regular surface. Overall, we conclude that surfaces with irregular series of bars are perceived as intermediate stimuli between the smooth and the regular surfaces depending on the size of the widest smooth interval present.

\section{Rats use their whiskers to solve surface discrimination}

To assess the role of the whiskers during the regular versus smooth discrimination task, we trimmed all the macrovibrissae on both sides of the snout and measured the resulting effect on performance. After cutting the whiskers, the performance dropped significantly compared with the previous control session (Fig. $4 A, B$, paired $t$ test, $p<10^{-4}$ ). More precisely, the performance was at chance level during the first third of the session (Fig. $4 A$, binomial test, $p>0.1, n=6$ rats) and then increased, although not to the same level as before cutting. A second test session confirmed the drop in performance after whisker trimming (Fig. $4 A$, right). The decrease in performance indicates that the rats learned to solve the task using their macrovibrissae. The partial recovery afterward further indicates that they can find an alternative strategy rather quickly during the first session after
A

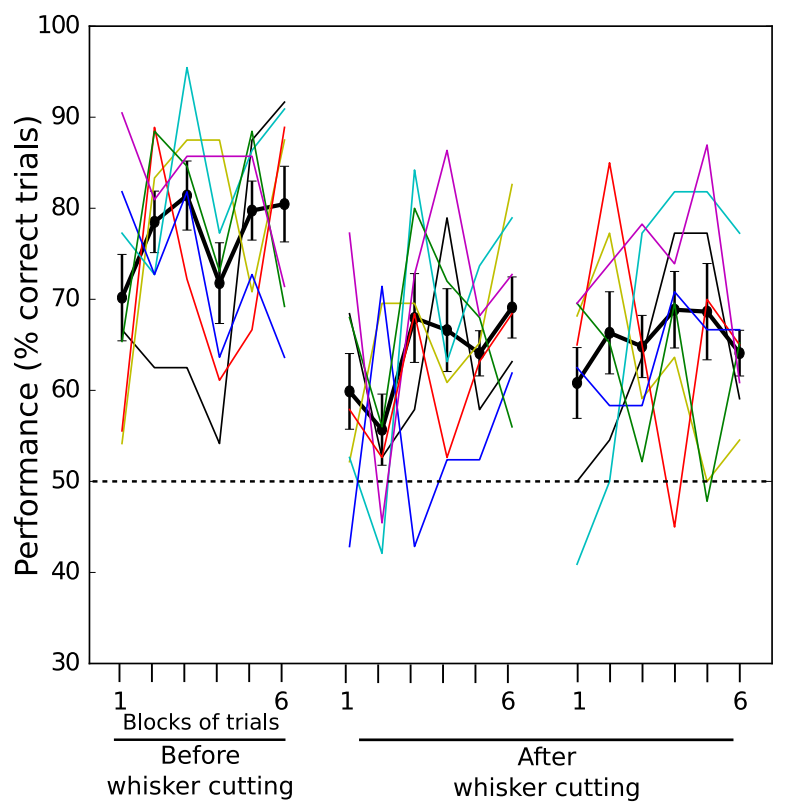

B

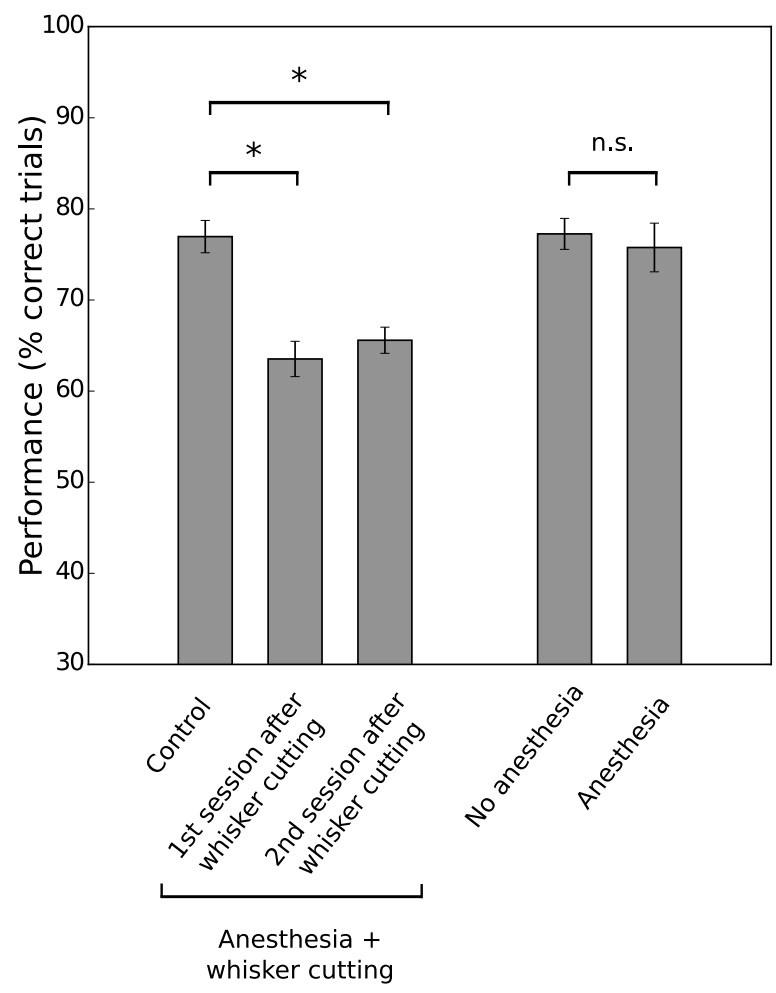

Figure 4. Whisker cutting impairs discrimination. $\boldsymbol{A}$, Performance for the session before and two sessions after whisker cutting ( $n=7$ rats). Each session (regular vs smooth) has been split into six blocks of trials. $\boldsymbol{B}$, Performance averaged over all trials of the sessions before and after whisker cutting ( $n=7$ rats) and before and after anesthesia only ( $n=3$ rats). ${ }^{*} p<0.05$, paired $t$-test.

whisker removal. We also investigated whether the initial reduction in performance could be a consequence of the brief anesthesia used for whisker cutting. Three control animals were anesthetized using the same procedure but without cutting the whiskers. They showed no drop in performance (Fig. $4 B$, paired $t$ test, $p>0.05$ ), excluding the possibility that anesthesia was causing the reduction in performance after whisker trimming. 
Interestingly, the rats could still navigate the maze after cutting the whiskers, even though nontactile cues were tightly controlled (see Materials and Methods). There was no change in the mean time to complete a trial $(11.1 \pm 1 \mathrm{~s}$ vs $11.8 \pm 1.2 \mathrm{~s}$ before and after cutting, paired $t$ test, $p=0.1, n=7$ rats). The long training on the maze up to the whisker cutting, taking 4 months or more, combined with remaining tactile information from the rest of the body, including the paws, were enough for the animals to navigate very rapidly. We hypothesized further that the partial recovery of discrimination performance during sessions without macrovibrissae could depend on tactile cues from the microvibrissae, the head, or the trunk. To test this possibility, we analyzed two sessions recorded by videography just after whisker cutting. We measured the minimal distance between the rat snout and the stimuli by manual tracking. We found that it was $>8 \mathrm{~mm}$ in at least $95 \%$ of the trials. This indicates that it is unlikely that the rats used their microvibrissae to discriminate the stimuli. Touching of the stimuli with the head fur remained possible in $\sim 15 \%$ of the trials (20/116 and 19/142 trials for the two rats; see Materials and Methods). We conclude that rats use their whiskers to solve the task in normal conditions, although they can learn another strategy if needed, which could involve the skin on the head posterior to the whisker pad as well as on the rest of the body.

\section{Neural activity in the barrel cortex is involved in solving the task}

To test the involvement of the somatosensory cortex during the regular versus smooth discrimination, we used the GABA-A agonist muscimol to inactivate the $S 1$ cortex while the animals were performing the task $(n=3)$. First, we determined the temporal window within which the cortex, but not the subcortical regions, could be inactivated when applying muscimol on the cortical surface. We recorded neuronal activity extracellularly using tetrodes in the barrel cortex and, as a control, in the hippocampus before and after a topic application of muscimol on S1 in rats anesthetized with isoflurane (acute experiments, $n=2$ animals). We found that $200 \mu \mathrm{l}$ of muscimol at $0.5 \mathrm{mg} / \mathrm{ml}$ inactivated the S1 cortex after $40-50 \mathrm{~min}$ for a period of at least $3 \mathrm{~h}$. Indeed, multiunit activity in response to deflection of a whisker decreased dramatically after muscimol application (Fig. 5A; 2 recordings followed up to $3 \mathrm{~h}$ were still at $0 \mathrm{sp} / \mathrm{stim}$ ). On the contrary, the hippocampus was still active after $3 \mathrm{~h}$ : spontaneous activity was equal to 33 spikes/s (sp/s) 10-20 min before muscimol application and to $14 \mathrm{sp} / \mathrm{s} 170-180 \mathrm{~min}$ after. Based on these results and on the literature (Higley and Contreras, 2006), rats already trained on the regular versus smooth protocol were fitted with custom-made implants containing two reservoirs, one over each S1 cortex, initially filled with saline (see Materials and Methods). The behavioral tests were done at least $48 \mathrm{~h}$ after the surgery. During bilateral inactivation of the S1 cortex with muscimol, the mean performance of the regular versus smooth discrimination task significantly decreased from $73.3 \pm 1.4 \%$ (control) to $58.8 \pm$ $2.3 \%$ (mean \pm SD; Fig. $5 B, C$ ). By looking at the time course within the session, we found that the percentage of correct trials remained at chance level during the first half and then increased back during the second half (Fig. $5 \mathrm{~B}$ ). One day after the muscimol test, we applied saline $(n=2)$ or no solution $(n=1)$ and found that the performance was immediately back to the control level (Fig. $5 B, C$, paired $t$ test, $p>0.05$ ). The running speed and the number of trials per session were not different between muscimol and control conditions (paired $t$ test, $p>0.05$ ) and there was no sign of ataxia (Sturgeon et al., 1979), suggesting that the general
A

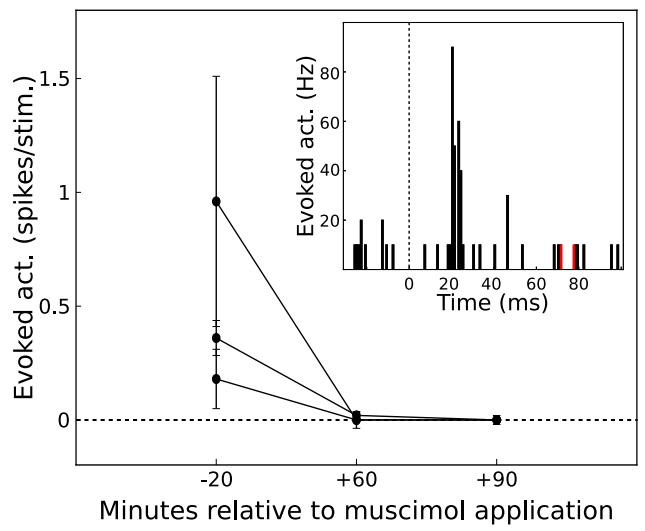

B

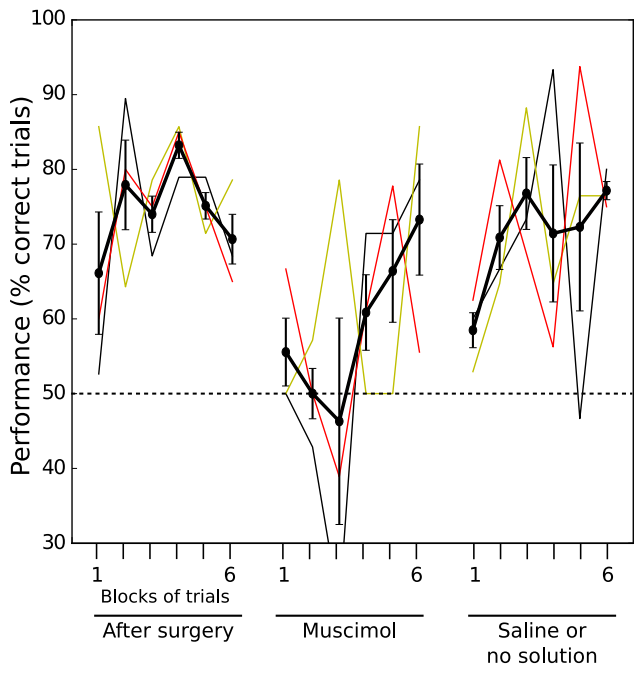

C

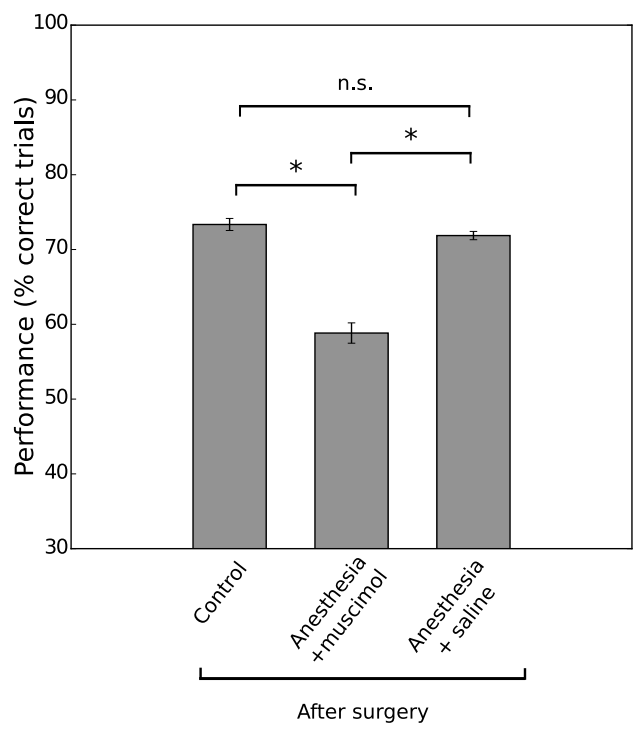

Figure 5. Cortical inactivation impairs discrimination. $\boldsymbol{A}$, Multiunit activity in response to stimulation of a functionally identified whisker as a function of time around the application of muscimol on the barrel cortex surface ( $n=3$ recordings in 2 rats). Inset, Peristimulus time histogram of the activity recorded on one tetrode while stimulating whisker D2 20 min before (black) and $60 \mathrm{~min}$ after (red) muscimol application. Activity $90 \mathrm{~min}$ after was null. $\boldsymbol{B}$, Performance during a control session, during muscimol application, and during saline application (two rats) or no solution (one rat). Each session (regular vs smooth) has been split into six blocks of trials. C, Performance averaged over all trials of the sessions before and after muscimol or saline cortical application ( $n=3$ rats). ${ }^{*} p<0.05$, paired $t$-test. 
behavior of the rat was not affected by the muscimol application. We analyzed one video recording by manual tracking of the head. Results were similar to those obtained after whisker cutting, with no evidence for microvibrissae touch and limited involvement of the head skin (see above). Overall, these results indicate a role for the somatosensory cortex in the discrimination of tactile patterns, with partial recovery in time that could be mediated by distant body parts.

\section{Running speed in the central alley}

To estimate whether the rats could be sampling the stimuli several times by whisking on them, we first measured the running speed in the central alley of the maze based on the two IR detectors placed before and after the main stimulus site (Fig. 1A). As already stated, after learning, the mean running speed was $117.9 \pm 7.6 \mathrm{~cm} / \mathrm{s}$ (mean $\pm \mathrm{SD}$ ). Given that the stimulus was 102 $\mathrm{mm}$ long, we infer that the mean contact duration between the whiskers and the stimuli is $\sim 86 \mathrm{~ms}$, which is less than a typical whisking cycle at $10 \mathrm{~Hz}$. This result strongly suggests that the rats do not whisk on the stimuli.

The running speed measure also allowed us to investigate its possible effect on performance. Indeed, it has been shown that the duration of whisker contact is a critical parameter for discrimination (Zuo et al., 2011) and also that the scanning speed modulates the amplitude of vibrissae micromotions (Ritt et al., 2008). We found that the running speed was significantly higher for successful compared with failed trials, although the size of the effect was quite small (respectively, $118.8 \pm 7.8 \mathrm{~cm} / \mathrm{s}$ and $115 \pm$ $9.1 \mathrm{~cm} / \mathrm{s}$, Wilcoxon test, $p<9 \times 10^{-7}$, mean $\pm \mathrm{SD}$ calculated over 20 sessions or $\sim 2400$ trials after learning, $n=5$ rats). This result suggests that there may be an optimal speed for tactile scanning of the stimuli.

Whiskers retract slowly at the estimated time of first contact To gain more insight into a possible strategy of the animals regarding the positioning and dynamics of the whisker arrays during the task, we placed IR reflectors on the $\mathrm{C} 1$ or $\mathrm{C} 2$ whiskers to track the angle on the left side $\left(\theta_{L}\right)$ and on the right side $\left(\theta_{R}\right)$ of the snout while the rats were approaching and contacting the stimuli (Fig. $1 B, n=15$ regular vs smooth sessions on 3 rats). Addition of such a lightweight marker (1-2 mg) was reported to not alter whisker kinematics (Bermejo et al., 1998). Analysis of these video recordings provided further evidence on the absence of whisking during contact with the stimuli. Figure $6 A$ shows the left and right whisker angle as a function of time during a typical discrimination trial. The time course was slow, with no evidence for oscillatory movements. In contrast, we were able to find an example of spontaneous whisking with clear oscillations at $\sim 10 \mathrm{~Hz}$ (Fig. 6B). Power spectrum calculation confirmed that there was very little signal in the $5-12 \mathrm{~Hz}$ whisking-frequency band during the discrimination periods (Fig. 6C; Jenks et al., 2010). We conclude from these results that rats did not discriminate the stimuli by whisking on them.

Next, we investigated whether the rats were approaching the stimuli with random whisker angle and speed or if these variables were actively controlled before and/or during the discrimination. The eyes reflected IR beams (Fig. 1B), so we could obtain the head angle $(\gamma)$ relative to the central alley axis and the whisker angles relative to the head axis (Fig. $1 C$ ). In this configuration, a protracted state for the whiskers is defined by an angle $>90^{\circ}$. Figure $7 A$ shows the angular trajectory of the right whisker as a function of the position of the rat eyes in the central alley for the 665 trials of one animal (five sessions). The red horizontal line indicates the
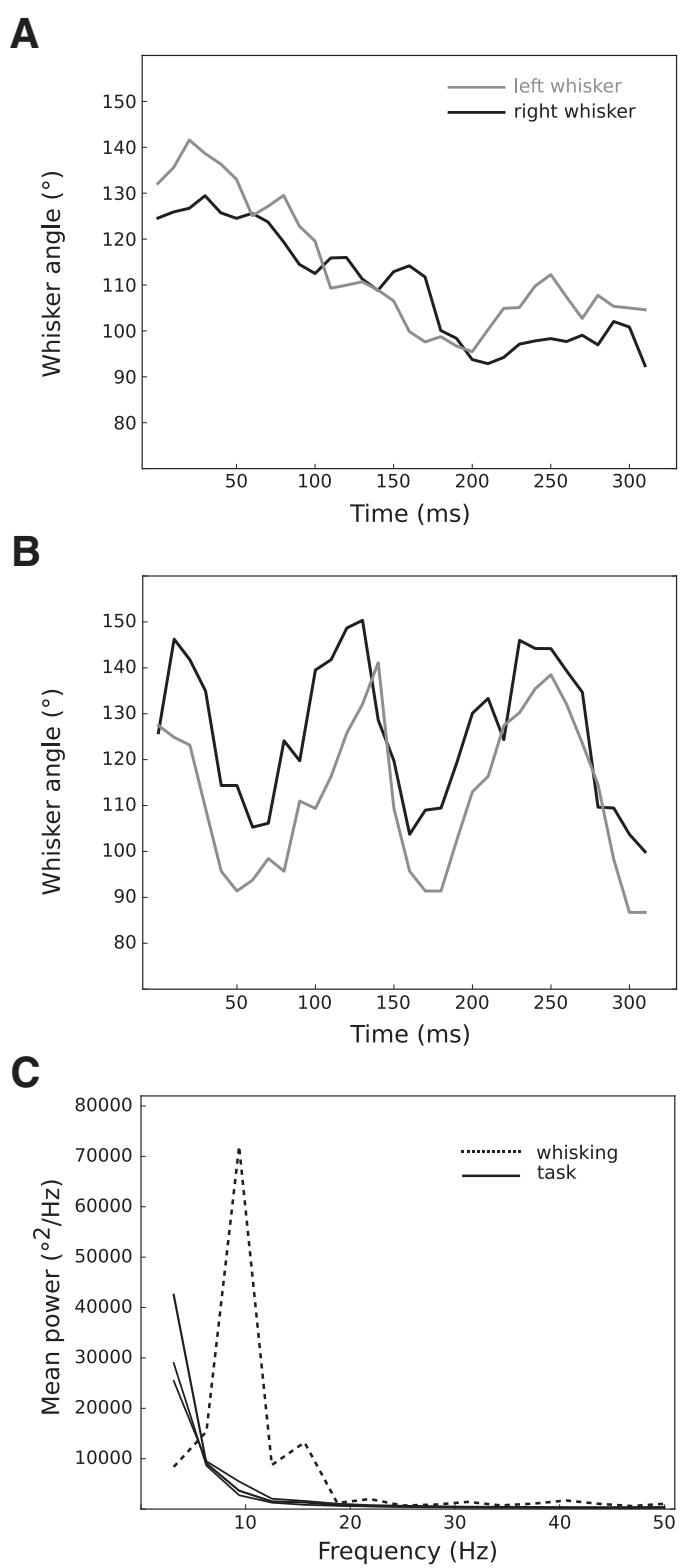

Figure 6. Discrimination of stimuli was performed without whisking. $\boldsymbol{A}$, Left and right whisker angle as a function of time for an example discrimination trial in the central alley. $\boldsymbol{B}$, Left and right whisker angle as a function of time during a spontaneous bout of whisking in a reward arm. C, Power spectrum of the whisker angle ( $n=336$ trials, 3 rats) and power spectrum of the traces of $\boldsymbol{B}$ showing a peak in the whisking frequency range.

physical position of the stimulus in the alley and the black arrow the estimated position where the rat would first be able to contact the stimulus if it had its whiskers maximally protracted. Two consecutive points on each colored curve are separated by $10 \mathrm{~ms}$. We observed that the whisker followed a reproducible trajectory from trial to trial. It was initially protracted when approaching the stimulus, with an angle of $121 \pm 7.9^{\circ}$ (mean \pm SD) $90 \mathrm{~ms}$ before the first possible contact, and then retracted to $99.2 \pm 8.1^{\circ}$ (mean $\pm \mathrm{SD}$ ) $170 \mathrm{~ms}$ after the first possible contact (Fig. $7 A$ ). It is important to note that the markers were placed on the tip of whiskers cut $8-10 \mathrm{~mm}$ from the skin of the snout so that the markers could not touch the stimuli. This implies that the measured movement of the marker was not due to the physical contact onto the stimulus, but rather to the movement of the whisker itself. Therefore, the whisker angle values that we measured re- 
A

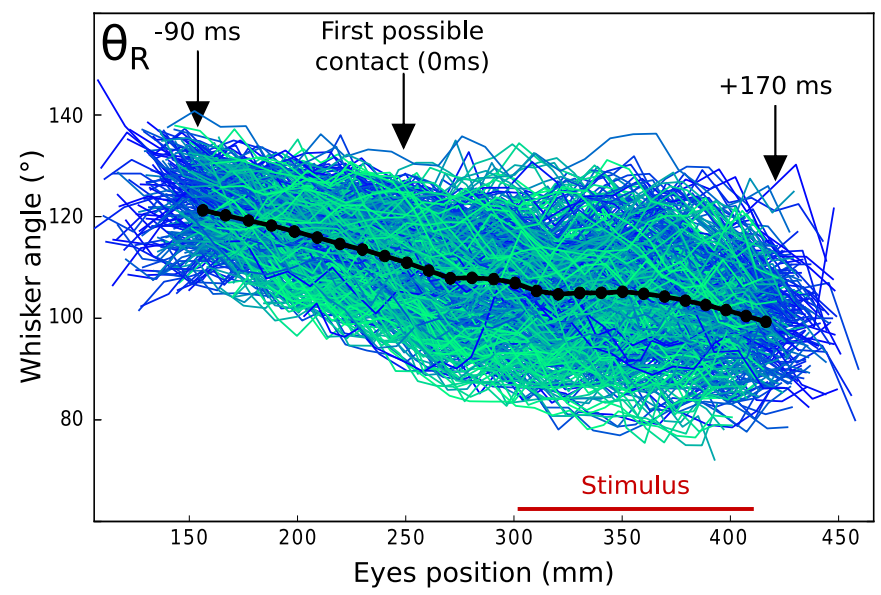

B

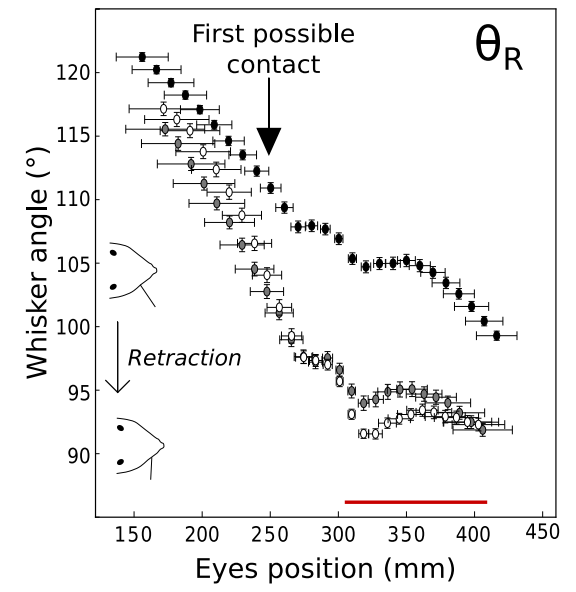

C

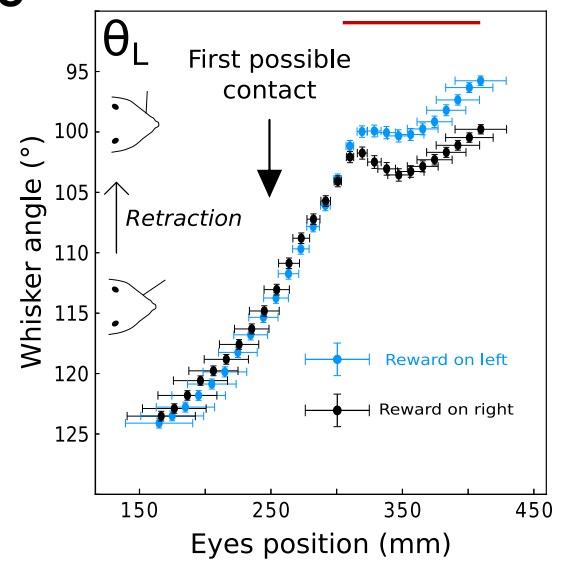

D

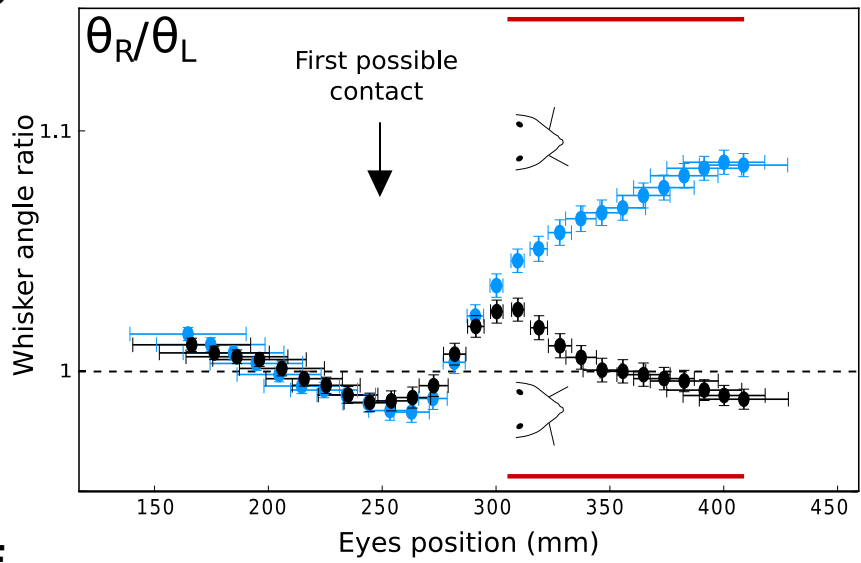

E

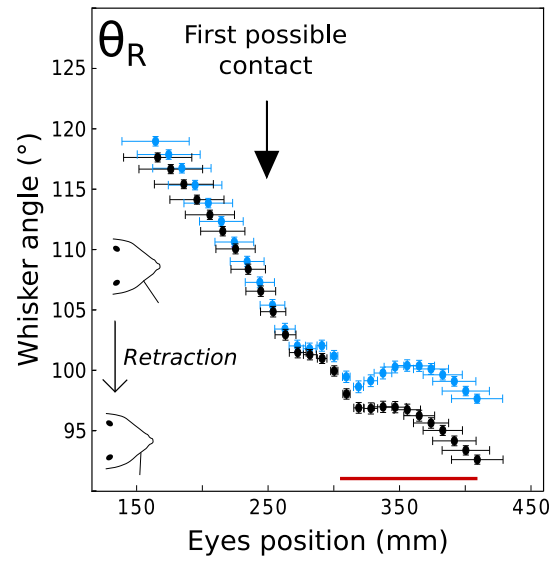

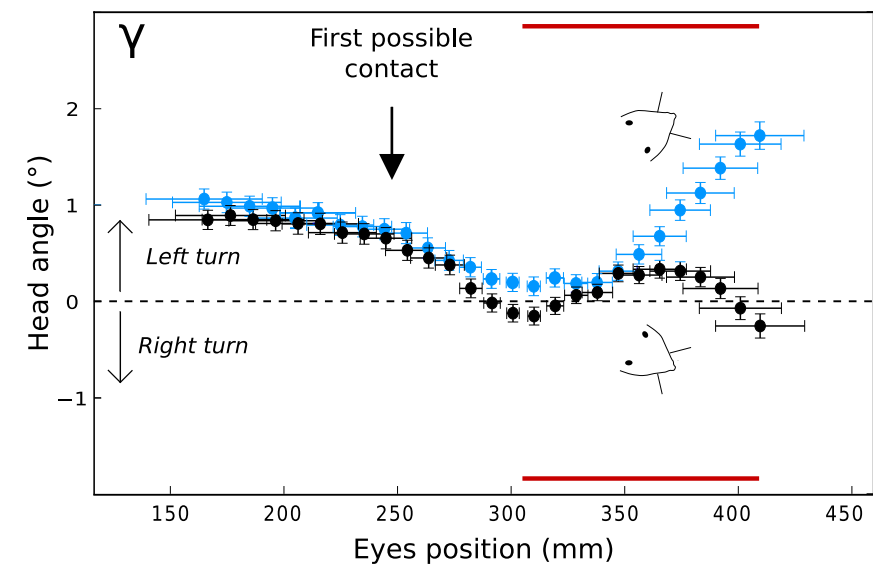

Figure 7. Whisker angle and head direction during stimulus discrimination. $A$, Individual trial trajectories of the right whisker angle as a function of the rat position (measured as the eyes position) in the central alley for five regular versus smooth sessions in the same animal ( $n=665$ trials). The color indicates time within sessions (green: beginning; blue: end). Each point corresponds to the measure on one video frame; 27 frames were analyzed for each trial. Interval between two points was $10 \mathrm{~ms}$. The thick black line indicates the mean trajectory. The red horizontal line indicates the physical position of the stimulus along the alley and the vertical arrow indicates the first possible contact between the whiskers and the stimulus if the whiskers were fully protracted. $\boldsymbol{B}$, Mean $( \pm$ SEM) right whisker angular trajectories for each of three rats as a function of the rat position in the central alley. The horizontal error bars represent the SD of the eye position distribution. For panels $\boldsymbol{B}-\boldsymbol{E}$, only successful trials were kept. C, Left (top) and right (bottom) whisker angle values as a function of the rat position in the central alley ( $n=3$ rats). In each panel, the angle values have been separated in two groups depending on whether the rewarded stimulus was on the right (black trace) or on the left (blue trace). $\boldsymbol{D}, \boldsymbol{E}$, Whisker angle ratio (right/left) and head direction plotted as a function of the rat position in the central alley $(n=3$ rats). Angle ratios have been divided by their baseline value before averaging across animals.

port the general protraction state of each vibrissal array. Similar whisker trajectories were observed for the three rats (Fig. $7 B$ ), confirming that the animals developed a particular motor strategy to explore the stimuli.

\section{Orientation of the whiskers and head direction during} the task

Given the reproducibility of the whisker angular trajectory while the animals were engaged in the discrimination, we wondered 
whether we could observe a relation between the stimulus encountered and the ongoing whisker movement. The animals might adjust their touch dynamically depending on whether they recognize the rewarded or an unrewarded stimulus. We found that, during successful trials, the whisker protraction level on both sides of the snout was modulated by the side of the rewarded stimulus. More precisely, the right whisker retracted more when the rewarded stimulus was placed on the right (Fig. $7 C$, top, black trace) than when it was placed on the left (blue trace). Conversely, the left whisker retracted more when the rewarded stimulus was placed left (Fig. 7C, bottom). Therefore, during successful trials, the whisker position was actively modulated as a consequence of the position of the rewarded stimulus. To quantify whisker asymmetry, we calculated the ratio between the right and left whisker angles $\left(\theta_{\mathrm{R}} / \theta_{\mathrm{L}}\right)$. An increase in this ratio indicates that the whiskers are orienting to the left side (Fig. 1C). The results are shown in Figure $7 D$. After the first possible contact, the mean ratio value increased when the rewarded stimulus was on the left side and decreased when the rewarded stimulus was on the right side. Therefore, during successful trials, the whisker arrays were adjusted by the animal and oriented toward the rewarded stimulus. The mean ratio value was significantly higher for left-rewarded trials compared with right-rewarded trials as soon as $60 \mathrm{~ms}$ after the first possible contact (Wilcoxon rank-sum test, $p<1.9 \times$ $10^{-3}$; Fig. $7 D$ ), indicating that this dynamic adjustment was taking place already well before the end of contact with the stimulus. We verified that the excess retraction on the rewarded stimulus was not due to friction on the rewarded surface, always rougher than the nonrewarded smooth surface, by analyzing failed trials. In those instances, the whisker arrays did not orient toward the regular bars surface after the initial contact. If anything, there was a tendency for transient excess retraction on the smooth surface. This observation leads us to conclude that, in successful trials, the orientation of the whisker array toward the rewarded stimulus is not a mechanical effect, but rather an active process. In addition, we observed that the animal started to turn its head toward the rewarded stimulus side $\sim 130 \mathrm{~ms}$ after the first possible contact (Wilcoxon rank-sum test, $p<4.2 \times 10^{-3}$; Fig. $7 E$ ). We conclude from these data that rats running at high speed in a narrow alley can discriminate patterned stimuli placed on the sides within single sweeps and that they anticipate the turn indicating their choice by first orienting their whiskers and then orienting their head toward the rewarded side.

\section{Discussion}

We developed a new two-alternative forced choice task that involves fast $(<100 \mathrm{~ms})$ and simultaneous discrimination of surfaces with or without vertical regularly spaced bars. The rats learned to distinguish these surfaces in 8 weeks and could further distinguish patterns of vertical bars differing in their spatial arrangement. Learning of the task involved the use of the whiskers and neural activity in S1. Nonetheless, alternative strategies to solve the task were rapidly developed when the whiskers were cut or when S1 was inactivated. Rats performed the discrimination while running at full speed without whisking, but with precise and systematic control of the whisker arrays. During successful trials of the task, the whiskers were actively positioned in an asymmetric manner around the snout and oriented toward the rewarded stimulus. This whisker asymmetry was followed by a turn of the head toward the rewarded stimulus $70 \mathrm{~ms}$ later as the rat was leaving the stimuli site.

\section{Reminders of the rewarded stimulus guide learning}

The idea of stimulus reminders placed between the discrimination site and the reward site to guide and accelerate learning was inspired from a recent visual discrimination task study (Harvey et al., 2012). The goal was to shorten the delay between the last contact with the rewarded stimulus and the reward and thus to facilitate the association between them. In our study, all the rats quickly learned the task with two reminders (stage 1; Fig. 2A). Interestingly, the removal of the reminder at the choice site reduced the performance to chance level, indicating that the rats were basing their discrimination largely on these stimuli when present. Indeed, further learning in the task was slow. One way to speed up learning in future experiments could be to transition slowly between stages 2 and 3 by keeping the reminders at the choice site during $\sim 80 \%$ of the session and decrease this proportion gradually once learning becomes measurable.

\section{Rats can discriminate regular from irregular bar series}

When we introduced surfaces with irregular series of bars, the rats showed immediately a very high performance in discriminating the I13 surface from the smooth surface, with no significant decrease compared with the regular versus smooth discrimination level. Two hypotheses could be raised: either they do not perceive the difference between the regular and irregular surfaces or they quickly associate them. Given that the direct regular versus irregular combination dropped performance down to chance level, one could infer that the animals are not able to perceive the difference between the two series. However, many reports in the literature already highlighted the abilities of rats to discriminate fine differences between two surfaces (Carvell and Simons, 1990, 1995; von Heimendahl et al., 2007; Morita et al., 2011). In particular, rats can discriminate spaces between grooves that differ by $125 \mu \mathrm{m}$ (Carvell and Simons, 1995), suggesting that they could learn to perceive the difference between the bar series in our study. Therefore, we think that the animals learned to associate only the presence of the bars with the reward, not the fact that these bars were displayed regularly. This contingent generalization is likely a consequence of the task design based on two-alternative choices. Nonetheless, by varying the size of the maximal smooth interval in the irregular series from 13 to $50 \mathrm{~mm}$, we revealed that the irregular surface could be distinguished from the regular surface during acute comparisons. In particular, when a long smooth interval was present, the regular versus irregular discrimination became similar to the regular versus smooth discrimination. We conclude that the animals are able to base their discrimination on the pattern of the bars on the surface and that the precise location of the bars governs the difficulty of the discrimination.

\section{Whisker cutting impairs task performance}

To ensure that the discrimination task involves the whiskers, we cut all macrovibrissae ( $n=7$ rats). We found that the performance of the animals immediately decreased to chance level, confirming the involvement of the whiskers. However, maze navigation was not altered and the performance increased back within one session, suggesting that the animals may find quickly an alternative strategy. This recovery could be based on tactile inputs conveyed by receptors other than those of the macrovibrissae. We were controlling the visual, auditory, and olfactory conditions by training animals in total darkness, constant masking noise, and wiping stimuli with ethanol $30 \%$ so that we could exclude the use of nontactile cues. We thus speculate that the rats learned to use the skin of their head or trunk. Indeed, it was shown previously that rats are able to discriminate surfaces 
using their microvibrissae (Brecht et al., 1997; Kuruppath et al., 2014) or even the skin of their snouts (Morita et al., 2011). However, we tracked the animal head manually on the video recordings of sessions for two rats after whisker cutting and found that the rats never stopped on the stimuli. Instead, in at least $95 \%$ of the trials, the rats ran rather parallel to the stimuli without approaching the nose and hence the microvibrissae. We conclude that intact rats readily discriminate stimuli placed on their path using their whiskers. In addition, they can develop a new strategy if their whiskers are removed, probably based on tactile inputs from the skin either on the posterior part of the head or on the trunk.

\section{Cortical inactivation reduces behavioral performance}

We tested the effects of cortical inactivation by applying muscimol on the surface of the barrel cortex during the behavioral session using a custom-made bilateral implant. Performance decreased to chance level immediately at the start of the session even though, as for whisker-cutting experiments, maze navigation was normal and no ataxia could be detected (Sturgeon et al., 1979). Cortical inactivation was in fact even more effective than whisker cutting: in the first half of the sessions, the performance was lower after muscimol than after whisker cutting for each of the three rats tested in both (mean value $50.8 \%$ vs $60.6 \%$ ), and the recovery took longer to occur (cf. average performance in middle session, Figs. $5 B, 4 A$ ). This is not necessarily surprising because muscimol could have spread outside of the barrel cortex region, invading part of the trunk region juxtaposed next to it. This result strengthens the idea that the rats could use the skin of their trunk as an alternative strategy to using their whiskers.

\section{Whisker contact on surfaces is tightly controlled throughout the discrimination}

Whisking is a sampling strategy often displayed by rodents performing roughness discrimination and it can be adjusted quickly and accurately during tasks (Harvey et al., 2001; Voigts et al., 2015). Whisking has two main consequences that could be beneficial to surface perception: it allows the whiskers to contact the discriminanda several times before making a choice and it sets a particular speed of the whiskers on the scanned surface. We designed the task such that multiple sampling would not be favored because it would lengthen trials and thus prevent receiving rewards frequently. Indeed, in our protocol, the rats could be trained to perform the task at high running speed without stopping and coming back after first encountering the stimuli so that the whiskers contacted the surfaces only once during each trial. To confirm that this single contact event did not involve whisking, we first estimated its duration. The running speed of the animal was $\sim 1 \mathrm{~m} / \mathrm{s}$. This value is at the higher end of the range of speeds observed on freely moving animals exploring surfaces, between 0.2 to $0.6 \mathrm{~m} / \mathrm{s}$ (Carvell and Simons, 1990; Ritt et al., 2008; Grant et al., 2009) up to $1.2 \mathrm{~m} / \mathrm{s}$ (Hobbs et al., 2015). The resulting whisker-stimulus contact duration, estimated at $86 \mathrm{~ms}$, was too short to allow a whisking cycle. Second, we calculated the power spectrum of whisker movements and found no evidence for a peak in the whisking frequency band, but rather a gradual decrease in power as a function of frequency (Fig. 6). This is in contrast to a study by Jenks et al. (2010), who reported the presence of whisking in approximately one-third of trials while rats ran in a central alley. However, their animals were not trained to detect or discriminate stimuli and thus probably did not adopt a specific strategy regarding their whiskers. This is supported by the observation that, in contrast to our study, these animals did not approach the stimuli with a specific whisker angle (Jenks et al., 2010).

We conclude that rats are able to discriminate surfaces without whisking, passing their whiskers on the stimuli only once in a continuous sweep while running in an alley. We propose that the main behavioral advantage of whisking may be to impose a whisker-on-stimulus contact speed in an optimal range to ensure an efficient transformation of the surface profile into mechanoreceptor activation patterns (Boubenec et al., 2014). The fast active movement of whisking would not be necessary anymore when the animal is running at an adequate speed along surfaces, as in our study. This hypothesis is compatible with other studies arguing for active control of the contact speed, including the recent report that whisker speed is kept constant under wind perturbations (Saraf-Sinik et al., 2015). In addition, it does not preclude a role of whisking for multiple sampling in more complex object recognition tasks.

Finally, we observed that the rats controlled precisely the position of their whiskers before contacting the stimuli and further oriented them as early as $60 \mathrm{~ms}$ after the first possible contact. This whisker movement was followed by a turn of the head toward the same side while the rat was leaving the stimuli. These results agree and extend the head-turning asymmetry process previously described in goal-directed (Towal and Hartmann 2006; Schroeder et al., 2016) and spontaneous exploratory (Mitchinson et al., 2007) behaviors of rats and mice, reflecting a stimulus-driven attentional process (Mitchinson et al., 2013).

\section{References}

Bermejo R, Houben D, Zeigler HP (1998) Optoelectronic monitoring of individual whisker movements in rats. J Neurosci Methods 83:89-96. CrossRef Medline

Boubenec Y, Claverie LN, Shulz DE, Debrégeas G (2014) An amplitude modulation/demodulation scheme for whisker-based texture perception. J Neurosci 34:10832-10843. CrossRef Medline

Brecht M, Preilowski B, Merzenich MM (1997) Functional architecture of the mystacial vibrissae. Behav Brain Res 84:81-97. CrossRef Medline

Carvell GE, Simons DJ (1990) Biometric analyses of vibrissal tactile discrimination in the rat. J Neurosci 10:2638-2648. Medline

Carvell GE, Simons DJ (1995) Task- and subject-related differences in sensorimotor behavior during active touch. Somatosens Mot Res 12:1-9. CrossRef Medline

Grant RA, Mitchinson B, Fox CW, Prescott TJ (2009) Active touch sensing in the rat: anticipatory and regulatory control of whisker movements during surface exploration. J Neurophysiol 101:862-874. Medline

Guic-Robles E, Jenkins WM, Bravo H (1992) Vibrissal roughness discrimination is barrelcortex-dependent. Behav Brain Res 48:145-152. CrossRef Medline

Harvey CD, Coen P, Tank DW (2012) Choice-specific sequences in parietal cortex during a virtual-navigation decision task. Nature 484:62-68. CrossRef Medline

Harvey MA, Sachdev RN, Zeigler HP (2001) Cortical barrel field ablation and unconditioned whisking kinematics. Somatosens Mot Res 18:223227. CrossRef Medline

Higley MJ, Contreras D (2006) Balanced excitation and inhibition determine spike timing during frequency adaptation. J Neurosci 26:448-457. CrossRef Medline

Hobbs JA, Towal RB, Hartmann MJ (2015) Spatiotemporal patterns of contact across the rat vibrissal array during exploratory behavior. Front Behav Neurosci 9:356. CrossRef Medline

Hutson KA, Masterton RB (1986) The sensory contribution of a single vibrissa's cortical barrel. J Neurophysiol 56:1196-1223. Medline

Jacob V, Estebanez L, Le Cam J, Tiercelin JY, Parra P, Parésys G, Shulz DE (2010) The Matrix: a new tool for probing the whisker-to-barrel system with natural stimuli. J Neurosci Methods 189:65-74. CrossRef Medline

Jadhav SP, Feldman DE (2010) Texture coding in the whisker system. Curr Opin Neurobiol 20:313-318. CrossRef Medline

Jenks RA, Vaziri A, Boloori AR, Stanley GB (2010) Self-motion and the 
shaping of sensory signals. J Neurophysiol 103:2195-2207. CrossRef Medline

Johnson KO, Hsiao SS (1992) Neural mechanisms of tactual form and texture perception. Annu Rev Neurosci 15:227-250. CrossRef Medline

Kleinfeld D, Ahissar E, Diamond ME (2006) Active sensation: insights from the rodent vibrissa sensorimotor system. Curr Opin Neurobiol 16:435444. CrossRef Medline

Krupa DJ, Matell MS, Brisben AJ, Oliveira LM, Nicolelis MA (2001) Behavioral properties of the trigeminal somatosensory system in rats performing whisker-dependent tactile discriminations. J Neurosci 21:5752-5763. Medline

Kuruppath P, Gugig E, Azouz R (2014) Microvibrissae-based texture discrimination. J Neurosci 34:5115-5120. CrossRef Medline

Mayrhofer JM, Skreb V, von der Behrens W, Musall S, Weber B, Haiss F (2013) Novel two-alternative forced choice paradigm for bilateral vibrotactile whisker frequency discrimination in head-fixed mice and rats. J Neurophysiol 109:273-284. CrossRef Medline

Mitchinson B, Prescott TJ (2013) Whisker movements reveal spatial attention: a unified computational model of active sensing control in the rat. PLoS Comput Biol 9:e1003236. CrossRef Medline

Mitchinson B, Martin CJ, Grant RA, Prescott TJ (2007) Feedback control in active sensing: rat exploratory whisking is modulated by environmental contact. Proc Biol Sci 274:1035-1041. CrossRef Medline

Miyashita T, Feldman DE (2013) Behavioral detection of passive whisker stimuli requires somatosensory cortex. Cereb Cortex 23:1655-1662. CrossRef Medline

Morita T, Kang H, Wolfe J, Jadhav SP, Feldman DE (2011) Psychometric curve and behavioral strategies for whisker-based texture discrimination in rats. PLoS One 6:e20437. CrossRef Medline

O’Connor DH, Clack NG, Huber D, Komiyama T, Myers EW, Svoboda K
(2010) Vibrissa-based object localization in head-fixed mice. J Neurosci 30:1947-1967. CrossRef Medline

Paxinos G, Watson C (2009) The rat brain in stereotaxic coordinates, 6th ed. Amsterdam: Academic Press Elsevier.

Ritt JT, Andermann ML, Moore CI (2008) Embodied information processing: vibrissa mechanics and texture features shape micromotions in actively sensing rats. Neuron 57:599-613. CrossRef Medline

Sachidhanandam S, Sreenivasan V, Kyriakatos A, Kremer Y, Petersen CC (2013) Membrane potential correlates of sensory perception in mouse barrel cortex. Nat Neurosci 16:1671-1677. CrossRef Medline

Saraf-Sinik I, Assa E, Ahissar E (2015) Motion makes sense: an adaptive motor-sensory strategy underlies the perception of object location in rats. J Neurosci 35:8777-8789. CrossRef Medline

Schroeder JB, Ritt JT (2016) Selection of head and whisker coordination strategies during goal-oriented active touch. J Neurophysiol 115:17971809. CrossRef Medline

Sturgeon RD, Fessler RG, Meltzer HY (1979) Behavioral rating scales for assessing phencyclidine-induced locomotor activity, stereotyped behavior and ataxia in rats. Eur J Pharmacol 59:169-179. CrossRef Medline

Towal RB, Hartmann MJ (2006) Right-left asymmetries in the whisking behavior of rats anticipate head movements. J Neurosci 26:8838-8846. CrossRef Medline

Voigts J, Herman DH, Celikel T (2015) Tactile object localization by anticipatory whisker motion. J Neurophysiol 113:620-632. CrossRef Medline

von Heimendahl M, Itskov PM, Arabzadeh E, Diamond ME (2007) Neuronal activity in rat barrel cortex underlying texture discrimination. PLoS Biol 5:e305. CrossRef Medline

Zuo Y, Perkon I, Diamond ME (2011) Whisking and whisker kinematics during a texture classification task. Philos Trans R Soc Lond B Biol Sci 366:3058-3069. CrossRef Medline 